\title{
1. DAS LADISLAUS-VOKABULAR ROM, BAV, PAL. LAT. 1787 KODIKOLOGISCHE BESCHREIBUNG
}

\author{
Von Alois Haidinger
}

\subsection{Lagenaufbau, Beschreibstoff, Einband}

Die Handschrift besteht aus 67 Pergament- und sieben freien Papierblättern im Format $240 / 242 \times 165 \mathrm{~mm}$ und lässt sich folgendermaßen darstellen: $\mathrm{II}^{\mathrm{III}}+2^{\mathrm{V}}+\mathrm{I}^{2}+1^{3}+6 . \mathrm{V}^{63}+2^{67}$ $+(1+\mathrm{I})^{\Pi^{*}}$. Die ersten sechs und letzten drei Blätter sind aus Papier; das erste und letzte Folium dient jeweils als Spiegelblatt. Alle Papierblätter sind unfoliiert; die dem Buchblock (Blatt 1-67) vorangestellten Folia werden hier mit I-V bezeichnet, die nachgestellten mit I* und II*.

Das Vokabular ist in einen mit gelblichem Pergament überzogenen Pappeinband gebunden, der nach Schunke ${ }^{21}$ in Rom um 1780 entstanden ist. Der auf drei Bünde geheftete Buchblock ist stark beschnitten, der Fußschnitt gelb eingefärbt. In den Rückenfeldern findet sich die heute gültige Signatur sowie ein nur teilweise erhaltener Buchtitel ${ }^{22}$. Aus der Zeit dieser Neubindung stammen die quer eingebundenen drei Doppelblätter VorderdeckelSpiegel/Bl. I, Bl. II/BI. III und Bl. II*/Hinterdeckel-Spiegel. Bl. IV und V, die beiden Hälften eines Einzelblattes, waren hingegen ursprünglich wohl als Vorsatz und Nachsatz des Vorgängereinbandes in Verwendung ${ }^{23}$. Aus dieser Zeit dürfte auch das Einzelblatt I* stammen. Die Folien IV und I* weisen Wasserzeichen auf: einen 1541 in identischer Form nachweisbaren Adlerkopf ${ }^{24}$ auf Bl. IV, den Buchstaben P auf I*.

\subsection{Schrift}

Vokabular und Nachträge sind im 6. Jahrzehnt des 15. Jahrhunderts, innerhalb eines Zeitraums von wenigen Jahren, in kalligraphischer Bastarda und Vorfraktur niedergeschrieben worden. Der Grundstock des Vokabulars auf $4^{r}-65^{v}$, Z. 7, die Notiz über die Krönung

21 I. Schunke, Die Einbände der Palatina in der Vatikanischen Bibliothek, 3 Bde. (Studi e testi 216-218). Città del Vaticano 1962, speziell Bd. 2, S. 894. - 1581 war das Manuskript noch mit einem Ledereinband über Holzdeckeln versehen. (Vgl. die Eintragung im Inventar Rom, BAV, Pal. lat. 1939 auf 6r.)

${ }_{22}$ Im ersten Rückenfeld eine gedruckte Papieretikette mit handschriftlicher Signatur 1787 in roter Tinte in einem von Voluten gerahmten Feld, im zweiten Feld der Rest eines ehemals dreizeiligen Buchtitels in Tinte, noch erkennbar DICTI(ONARIUM...), im dritten Feld - wie auf dem Vorderdeckel-Spiegel - eine blaue Papieretikette mit Aufdruck Pal. lat. 1\%8\%.

${ }_{23}$ Die Blätter IV und 1 zeigen in ihrer Position völlig übereinstimmende Wurmlöcher, während das zwischen Bl. IV und 1 eingebundene Bl. V keinerlei Spuren von Wurmfraß aufweist. Terminus post quem für die Einfügung von Bl. V an dieser Stelle ist der Zeitpunkt der Anbringung des Bibliotheksstempels auf $1^{\mathrm{r}}$, da ein schwacher Abklatsch desselben auf der Versoseite von Bl. IV, jedoch nicht auf der unmittelbar anliegenden Versoseite von Bl. V sichtbar ist.

24 Das Adlerkopf-Wasserzeichens entspricht dem Zeichen Nr. 2211 bei C. M. Briquet, Les filigranes. Paris etc. 1907. 
des Königs Ladislaus (1453 X 25) auf $2^{\text {rv }}$ sowie die Nachricht vom Tod des Königs (1457 XI 23) auf $1^{\mathrm{v}}$ ist je einer - im folgenden mit den Siglen A, B und C gekennzeichneten - Hand zuzuweisen. Es folgt eine Übersicht der Texteinheiten in der Reihenfolge ihres Auftretens in der Handschrift:

$\left(1^{\mathrm{r}}\right)$ Gebete (Federproben) (Abb. 1): Schwach sichtbare Tintenlinierung. Vertikale Schriftraumbegrenzungen bis zu den Seitenrändern durchgezogen (Schriftraumbreite ca. $95 \mathrm{~mm}$ ). Zeilenlinierung nur zum ersten Gebet; von jener zum zweiten Gebet ist nur die oberste Schriftraumbegrenzung erkennbar. Die beiden in schwarzbrauner Tinte von Hand A eingetragenen Gebete brechen jeweils mit der fünften Zeile ab und sind daher als Federproben zu verstehen. Die Schrift des zweiten Gebetes ist bei engerem Zeilenabstand geringfügig kleiner (Schriftraumhöhe der Gebete $43 / 44 \mathrm{~mm}$ bzw. ca. $35 \mathrm{~mm}$ ).

$\left(1^{v}\right)$ Notiz vom Tod des Königs Ladislaus (Abb. 2): Der siebenzeilige Text wurde in der unteren Hälfte des Blattes dort eingesetzt, wo die Schrift der Gebete der Rectoseite nicht mehr durchschlägt. Es ist keine Linierung erkennbar, die Tintenfarbe ist dunkler als jene der vorangehenden und folgenden Texte. Geschrieben von Hand C.

$\left(2^{\text {rv }}\right)$ Notiz über Krönung des Ladislaus in Prag (Abb. 3 f.): Schriftraum $90 \times$ ca. $155 \mathrm{~mm}$ Tintenlinierung. Vertikale Begrenzungslinien bis zu den Seitenrändern durchgezogen. Auf der Rectoseite 19 Zeilen, auf der Versoseite hingegen nur die Linien für die ersten zwölf Zeilen durchgezogen. Zu Textende LADISLAUS REX in vergrößerten Ziermajuskeln. Geschrieben von Hand B. Anschließend ADCIP, die Initialen der Devise Amans Deum Clerum Iustitiam Pacem ${ }^{25}$ des Königs, in ca. zweizeiligen Goldlombarden von blauem Fleuronnéegrund.

$\left(3^{r}\right)$ Schriftbandtexte auf dem Devotionsbild des Johannes Holubař (Abb. 5). Die in roter Tinte eingetragenen Texte (Jhesu, filii Marie, qui plasmasti me, miserere mei etc. und Johannes Holubarz informator serenissimi principis regis Ladislai) sind ohne Zweifel von Hand C eingesetzt worden. Man vergleiche etwa die häufige Verwendung des z-förmigen $r$ sowie die Kürzungsstriche in Form breiter Haarstriche mit nach unten gebogenen Enden und einem mittig angesetzten kleinen Punkt oder Vertikalstrich.

$\left(3^{v}\right)$ Widmung in Goldschrift (Abb. 6). Schriftraum $57 \times$ ca. $95 \mathrm{~mm}$. Linierung in blauer Tinte. Sechs Zeilen in gegenüber dem Haupttext vergrößerter Schrift. Der Text bietet zu wenig Charakteristika, um eine eindeutige Zuordnung zu einer der im Vokabular vertretenen Hände zu erlauben. Denkbar wäre, daß er gleichzeitig mit der goldenen Filigranornamentik und eventuell auch von derselben Hand eingesetzt worden ist.

$\left(4^{r}-65^{v}\right)$ Vokabular (Abb. 7 f.): Schriftraum meist ca. $105 \times 145 / 150 \mathrm{~mm}$ zu 20 Zeilen auf Tintenlinierung. Linienschema: Vertikale Begrenzungslinien bis zu den Seitenrändern durchgezogen; erste Schriftzeile auf der zweiten Zeilenlinie; Schriftraum in drei Spalten unterteilt, die in der Regel von ca. $5 \mathrm{~mm}$ breiten Interkolumnien getrennt werden. Fast immer findet sich eine weitere Vertikalleiste in der Breite eines Interkolumniums: meist am rechten Schriftspiegelrand (z. B. $4^{\mathrm{v}}$ ), seltener nur am linken (z. B. $8^{\mathrm{r}}$ ); eine zusätzliche Vertikalleiste bisweilen auch an beiden Seiten des Schriftspiegels (z. B. $8^{v}$ ). Dasselbe Linienschema findet sich auch noch auf $66^{\mathrm{r}}$; auf $66^{\mathrm{v}}-67^{\mathrm{v}}$ sind hingegen nur Reste einer in den Proportionen mit den vorhergehenden Blättern übereinstimmenden Schriftspiegelrahmung erkennbar. Geschrieben in schwarzer Tinte von Hand A.

Mitunter wurde die Vorlage nicht zeilenweise abgeschrieben, sondern wurden - wie Abweichungen im Schriftduktus zeigen - Gruppen von lateinischen Begriffen auf einmal einge-

\footnotetext{
${ }_{25} \mathrm{Vgl}$. S. 26 A. 61
} 
setzt (z. B. auf $4^{r}$ ). In der jeweils ersten Schriftspiegelzeile zu Beginn der zweiten und dritten Spalte häufig vergrößerte Anfangsbuchstaben. Die bis zu drei Zeilen großen Majuskeln teils in Cadellenform ${ }^{26}$ (z. B. $7^{\mathrm{r}}, 15^{\mathrm{v}}, 45^{\mathrm{v}}$ ) und bisweilen mit Gruppen von drei Querstrichelchen verziert (z. B. $26^{r}$ ). In der jeweils letzten Schriftspiegelzeile öfter schlaufenähnliche Haarstrich-Ausläufer (z. B. $7^{\mathrm{v}}, 8^{\mathrm{v}}, 42^{\mathrm{v}}, 46^{\mathrm{v}}, 48^{\mathrm{r}}$ ).

Trotz geringfügiger Duktusschwankungen dürfte der Text mit Ausnahme der letzten sieben Zeilen auf $65^{\mathrm{v}}$ von einem einzigen Schreiber (Hand A) eingetragen worden sein. Die Buchstabenformen stimmen mit jenen der Hand B überein (beispielsweise findet sich Minuskel-r - etwa auf $4 \mathrm{r}$ - wie bei Hand B häufig in z-Form), die Satzmajuskeln sind allerdings flüchtiger ausgeführt und weniger vielfältig. Die für Hand B charakteristischen Kürzungsstriche und Zeilenschlusszeichen finden sich bei Hand A nicht: Auf Kürzungen wurde wohl zu Gunsten der Lesbarkeit des Vokabulars verzichtet, Zeilenschlusszeichen sind auf Grund der Textanordnung des Vokabulars überflüssig. Die letzten sieben Zeilen (Abb. 8) stammen von Hand C, wobei die letzte der Zeilen auf Grund der dunkleren Tintenfarbe und des leicht abweichenden Schriftduktus nicht gleichzeitig mit den übrigen eingesetzt worden sein dürfte. (Zum Schreiber C vgl. auch S. 33.)

Kreuztituli, Devisen: Der Kreuztitulus INRI auf $66^{\mathrm{v}}$ (Abb. 10) und 67 (Abb. 11) (in beiden Fällen wird der Schrägbalken des N spiegelverkehrt eingesetzt) sowie die Devise ADCIP auf $1^{\mathrm{v}}$ (Abb. 2) und 3r (Abb. 5) stammen - wie die übereinstimmende Form des mit kleinen Punkten besetzten I nahelegt - wahrscheinlich von einer einzigen Hand. Daß der Kreuztitulus auf $66^{\mathrm{v}}$ gleichzeitig mit dem IHS-Monogramm ausgeführt wurde, wird durch die übereinstimmende graubraune Tintenfarbe belegt; jener auf $67^{\mathrm{r}}$ wurde hingegen in tiefschwarzer Tinte wohl gleichzeitig mit der Devise ADCIP und dem größtenteils weggeschnittenen Eintrag (wahrscheinlich Johannes Holubarz...) rechts unten nachgetragen ${ }^{27}$.

\subsection{Buchschmuck}

Es folgt eine Beschreibung der Ausstattungselemente in chronologischer Reihenfolge:

Schlichte Lombarden werden für die Devise ADCIP auf $2^{v}$ und als Anfangsbuchstaben der lateinischen Begriffe des Vokabulars verwendet: zweizeilige Initialen in poliertem Gold für die Devise und die insgesamt zwanzig Textabschnitte des Vokabulars, alternierend in Rot und Blau gehaltene einzeilige für alle lateinischen Begriffe des Wörterbuches.

Auszeichnungspunkte in zwei deutlich unterscheidbaren Farbtönen (helles Gelb und schmutziges Ocker) dienen zur Hervorhebung der Anfangsbuchstaben fast aller Wörter der zweiten und dritten Spalte des Vokabulars (vgl. z. B. Abb. 8) sowie der Majuskeln in den Texten auf $1^{\mathrm{v}}-2^{\mathrm{v}}$ (Abb. 2-4). Auffällig ist, daß die Auszeichnungspunkte auf $2^{\text {rv }}$ sowie der deutschen und tschechischen Begriffe der jeweils ersten Schriftspiegelzeile bis $65^{\mathrm{v}}$ in Hellgelb gehalten sind und sich das Verhältnis in der Anzahl der hellgelben und ockerfarbenen Punkte pro Seite im Textverlauf zunehmend zu Gunsten der letzteren verschiebt. Die nach dem

${ }^{26}$ Zum Begriff „Cadellen“ vgl. H. KöLlner, Zur kunstgeschichtlichen Terminologie, in: Zur Katalogisierung mittelalterlicher und neuerer Handschriften. Frankfurt am Main 1963, 154.

${ }^{27}$ Knapp oberhalb des unteren Seitenrandes von $67^{\mathrm{r}}$ ist eine, die Oberlängen der genannten Inschrift kreuzende Tintenlinie erkennbar, die wohl als Rest eines Rechteckfeldes zur Aufnahme der Inschrift zu interpretieren ist. Die Ausdehnung der Linie legt nahe, daß die Inschrift mehr als den Namen des Informators enthalten hat; vermutlich war hier der selbe Text wie auf $3^{r}$ unten zu lesen. 
23. November 1457 eingetragene Notiz zum Tod des Ladislaus auf $1^{v}$ enthält ausschließlich ockerfarbene Punkte. Offensichtlich wurden die Texte in einem ersten Arbeitsgang nur lükkenhaft in Hellgelb ausgezeichnet, später, wahrscheinlich erst im Zuge der nach dem Tode des Königs erfolgten Ergänzung des Manuskriptes durch die Zeichnungen der „Holubař-Stilschicht", wurden die ockerfarbenen Auszeichnungspunkte eingesetzt. Keine Auszeichnungspunkte zeigen lediglich die als Federproben zu verstehenden Gebete auf $1^{\mathrm{r}}$ sowie die zum Zeitpunkt der Ausschmückung mit Auszeichnungspunkten noch nicht eingesetzten letzten sieben Zeilen des Vokabulars auf $65^{\mathrm{v}}$.

Fleuronnée in blauer Tinte findet sich im Binnengrund und als Besatz der die Abschnitte des Vokabulars einleitenden und die Devise auf $2^{\mathrm{v}}$ bildenden Goldlombarden ${ }^{28}$ (Abb. 4, 7, 25 f.). Nur die Initialen auf $4^{\mathrm{r}}$ und $7^{\mathrm{v}}$ bilden Ausläufer über die volle Blatthöhe aus, die übrigen Initialen zeigen hingegen nur relativ kurze, mit Perlenreihen besetzte Fortsätze.

Deckfarbenmalerei ist auf Bl. $3^{\mathrm{v}}$, die Widmungsseite des Wenzeslaus vom Elefanten mit einer Darstellung des Königs Ladislaus Postumus beschränkt (Abb. 6) ${ }^{29}$ : Die 83 × 90/95 mm messende Miniatur ${ }^{30}$ zeigt den jugendlichen König schräg nach links gewandt auf einem sockelartigen Thron, in den Händen Reichsapfel und Szepter. Das Bildfeld wird von einem plastisch abgestuften Rahmen in Altrosa gerahmt, dessen Ecken oben von den Wappen Ungarns und Böhmens, unten von jenen Österreichs und Luxemburgs belegt sind. In der Mitte des unteren Bildrandes befindet sich das Wappen Mährens. Der Hintergrund ist in Dunkelblau gehalten und mit einer weißen Federranke ornamentiert. Über dem Thron ist ein grünes, mit goldener Federranke gemustertes Tuch drapiert, auf dem Thron liegt ein blaugrauer Polster mit goldfarbenem Rankenmuster. Der König trägt über einem weißen Untergewand mit vor der Brust gekreuztem rosafarbenen Band einen karminroten, mit Goldornamentik verzierten, blau gefütterten Mantel und karminrote spitze Schuhe. Szepter, Krone, Reichsapfel und Mantelspange sind in Gold ausgeführt.

Der Rankenschmuck in den Freirändern ist im oberen Teil des Blattes als spiralig verlaufende goldene Fadenranken mit kugeligen, meist in Dreiergruppen auftretenden Endmotiven (als Binnenzeichnung stets zwei blaue Striche) sowie mit insgesamt sechs in Deckfarbenmalerei eingesetzten einfachen Blüten, im unteren Teil des Blattes hingegen als dichte, sich aus einer Blattmaske entwickelnde Blattranke in gelb gehöhtem Grün, kräftigem Blau, Rosa und Ocker ausgebildet. Die Deckfarbenranken sind mit verschiedenen Vögeln sowie von links nach rechts - mit Bär (?), Hirsch (?), Wildem Mann mit Stock, musizierendem Affen, Bär mit Kelchglas, Bogenschützen und springendem Hirsch belebt. Die Position des Stifterwappens, drei rote Flammen auf weißem Grund, sowie der unschön zusammengedrängte, die rechte Schriftraumbegrenzung wiederholt überschreitende Text sprechen dafür,

28 Goldlombarden mit Fleuronnée finden sich auf $2^{\mathrm{v}}, 4^{\mathrm{r}}, 7^{\mathrm{v}}, 8^{\mathrm{v}}, 12^{\mathrm{v}}, 15^{\mathrm{v}}, 18^{\mathrm{v}}, 21^{\mathrm{v}}, 22^{\mathrm{v}}, 23^{\mathrm{v}}, 26^{\mathrm{v}}, 28^{\mathrm{v}}, 31^{\mathrm{r}}, 32^{\mathrm{v}}$, $34^{\mathrm{r}}, 38^{\mathrm{v}}, 39^{\mathrm{r}}, 41^{\mathrm{r}}, 46^{\mathrm{r}}, 48^{\mathrm{v}}, 51^{\mathrm{v}}$.

29 Das Blatt besteht aus deutlich stärkerem Pergament als der Rest der Handschrift. Es wurde nach Ausführung der Deckfarbenranke und wohl vor Einsetzung des blauen Fleuronnées eingehängt: Auf der Rectoseite seines ca. $15 \mathrm{~mm}$ breiten, nach Bl. 2 sichtbaren Falzes ist die Spitze des Blattes oberhalb des springenden Hirschen auf $3^{\mathrm{v}}$ rechts unten zu sehen, die in blauer Tinte eingezeichnete Filigranornamentik auf $3^{\mathrm{v}}$ rechts oben ist wohl gleichzeitig mit dem Fleuronnéeschmuck der folgenden Seite nachgetragen worden.

30 Die Miniatur ist etwas schief auf dem Blatt plaziert und nicht exakt rechteckig: Abstand der Miniatur zum oberen Freirand 31-35 mm; Höhe der Miniatur 83, Breite oben 90, Breite unten 95 mm. 
daß der für den Widmungstext zur Verfügung stehende Raum durch bereits ausgeführte oder durch Vorzeichnung festgelegte Ranken begrenzt war. Wohl aus diesem Grunde ist das Wappen des Wenzeslaus vom Elefanten nicht in die Ranken an zentraler Stelle integriert worden, sondern mußte an einem von den Ranken noch nicht belegten Ort unmittelbar unterhalb des Textes untergebracht werden.

Die in Federzeichnung ausgeführten Darstellungen und Initialen des Vokabulars, auf die im folgenden unter dem Begriff „Holubař-Stilschicht“ Bezug genommen wird, sind erst nach dem Tod des Ladislaus im Zuge der Einfügung des Devotionsbildes des Johannes Holubař eingesetzt worden. Neben dem Widmungsbild auf $3^{r}$ gehören zu dieser Stilschicht die Tücherinitiale $\mathrm{O}$ zum ersten Gebet auf $1^{\mathrm{r}}$, das IHS-Monogramm auf $66^{\mathrm{v}}$, die Kreuzigung auf $67^{\mathrm{r}}$, das Tücheralphabet auf $65^{\mathrm{v}}-66^{\mathrm{v}}$ sowie die Fechtenden auf $67^{\mathrm{v}}$. Sie sind aus stilistischen Gründen einem einzigen Zeichner zuzuweisen. Auf Grund der Beobachtung, daß der Text der Schriftbänder im Widmungsbild auf $3^{r}$ von Hand C, dem Schreiber der Notiz vom Tod des Ladislaus, stammt, kann angenommen werden, daß die Zeichnungen wahrscheinlich etwa gleichzeitig mit der Niederschrift der Nachricht vom Tod des Königs eingesetzt worden sind. Dafür spricht auch, daß oberhalb der Fechtenden auf $67^{v}$ ursprünglich wohl eine zu $1^{v}$ gleichlautende Notiz über den Tod des Ladislaus eingetragen war. Umfang des getilgten Textes und noch einige erkennbare Textreste unterstützen diese Annahme. Wären die Fechtenden hingegen älter als diese Notiz, so hätte sie der Zeichner vermutlich nicht in die untere Hälfte sondern in die Mitte der Seite plaziert. Die Datierung 1474 auf $3^{r}$ kann - auch aus stilistischen Gründen - keinesfalls als Entstehungsjahr der Zeichnungen verstanden wer$\operatorname{den}^{31}$.

Die Devotionsdarstellung auf $3^{r}$ (Abb. 5) zeigt einen die Madonna mit ausgebreiteten Armen anbetender Ritter mit Helmzier und abflatternden Helmdecken. In der rechten oberen Bildecke, im von einer Wolkenkrause gerahmten Strahlenkranz, schwebt die Madonna als Dreiviertelfigur, das nackte Jesuskind auf der Linken, in der Rechten eine rote Frucht. In den beiden unteren Blattecken sind zwei - gegenüber dem knienden Ritter in kleinerem Maßstab gegebene - voll gerüstete Krieger mit geschlossenem Visier dargestellt. Um die diagonal ins Bild vorstoßende Lanze des Rechten windet sich ein Band mit der Inschrift Jhesu, filii Marie, qui plasmasti me, miserere mei etc. Zwischen den beiden Kriegern ist ein weiteres Schriftband: Johannes Holubarz informator serenissimi principis regis Ladislai, darunter die Jahreszahl 14\%4; am oberen Seitenrand die Devise ADCIP. Zwischen den beiden Schriftbändern ist ein Wappenschild mit drei Henkelkrügen plaziert, das völlig identisch auf $67^{\mathrm{r}}$ wiederbegegnet. Die Federzeichnung ist vor allem in kräftigem Rot und Hellblau koloriert; daneben wird schmutziges Gelb und helles Rosa verwendet: Rot zeigt sich für die Federn der Helmzier und einen Teil der Helmdecken, für den Wappenschild, für alle Inschriften und für einige Details der Rüstungen (Schuhe des Ritters und des linken Gerüsteten, Querriemen am rechten Oberschenkel desselben, Schwert des rechten Gerüsteten), Blau für die Wolkenkrause und das Untergewand Mariens sowie als Höhung für die Rüstungen, Hellrosa für das Obergewand Mariens und das Inkarnat der dargestellten Personen, Ocker für den von der Wolkenkrause umschlossenen Grund und - in einem kräftigeren Farbton - für die drei Henkelkrüge.

Die braun lavierte Tücherinitiale auf $1 r$ (Abb. 1) zeigt im Binnenfeld eine kleine nackte Figur des Jesuskindes; in seiner Linken hält es ein vertikal verlaufendes Schriftband mit der

\footnotetext{
${ }^{31} \mathrm{Vgl}$. S. 28 f.
} 
Aufschrift IHESUS, in der Rechten eine Frucht. Der Kopf des Jesusknaben entspricht völlig dem des Kindes auf $3 r$.

Das aus gefalteten und verflochtenen Bändern zusammengesetzte IHS-Monogramm auf $66^{v}$ mit einem kleinen Kruzifixus im Zentrum (Abb. 10) ist ebenso wie die dreifigurige Kreuzigung auf $67^{\mathrm{r}}$ (Abb. 11) in graubrauner Tinte laviert. Die Physiognomien der Assistenzfiguren stimmen vor allem in der Zeichnung der Augen mit der Maria auf $3^{r}$ sowie mit den beiden Schwertkämpfern auf $67^{v}$ (Abb. 12) überein. Letztere zeigen dieselbe, auf Hellblau, Dunkelblau, Rot und Ocker beschränkte Farbpalette wie die Devotionsdarstellung auf $3^{\text {r. }}$.

Das Tücheralphabet auf $65^{\mathrm{v}}-66^{\mathrm{v}}$ (Abb. 8-10) wurde teils auf linierten, eventuell für Nachträge vorgesehenen Seiten $\left(65^{v}\right.$ und $\left.66^{r}\right)$ nachgetragen. Es ist zweifellos jünger als die letzten sieben auf $65^{v}$ hinzugefügten Zeilen, andernfalls hätte der Zeichner auf $65^{v}$ mit Sicherheit zwei Zeilen des Tücheralphabets untergebracht. Das Alphabet ist aber auch jünger als das zur „Holubař-Stilschicht“ gehörende IHS-Monogramm auf $66^{v}$, denn die in unterschiedlicher Höhe angesetzten Buchstaben $T$ und $W$ nehmen offensichtlich Rücksicht auf das IHS-Monogramm. Die graublaue Lavierung der Buchstaben, die sich auch in den nach dem Tod des Ladislaus angelegten Zeichnungen auf $3^{\mathrm{r}}$ und $67^{\mathrm{v}}$ findet, spricht dafür, daß das Alphabet wohl unmittelbar nach Anlage der Zeichnungen der „Holubař -Stilschicht“ eingesetzt und gleichzeitig mit den Zeichnungen auf $3^{v}$ und $67^{v}$ laviert wurde. (Vgl. dazu auch die Anmerkungen zur programmatischen Ordnung des Kodex auf S. 27 f.)

\subsection{Verwandte Handschriften}

Im Zusammenhang ist es zweifellos notwendig und lohnend, das Augenmerk auf verwandte Handschriften zu lenken. Bereits Zahradnik hat auf Ähnlichkeiten der Schrift mit einem für König Ladislaus angefertigten Gebetbuch, heute Tepl, Stiftsbibl., Cod. 39, hingewiesen, diese jedoch nicht genauer spezifiziert ${ }^{32}$. Ruysschaert ${ }^{33}$ und kürzlich Metzger ${ }^{34}$ haben Teile des Buchschmucks der Werkstatt des sogenannten Lehrbüchermeister ${ }^{35}$ zugeschrieben; wohingegen der Autor auf die Verwandtschaft des Deckfarbenschmucks des Vokabulars mit jenem des Gebetbuches hingewiesen hat ${ }^{36}$.

Das in Tepl verwahrte Gebetbuch des Ladislaus hat in der Forschung weit mehr Beachtung als dessen Vokabular gefunden ${ }^{37}$. Es ist aus drei Teilen und vorgeschalteten Nachträgen

32 Vgl. I. Th. Zahradnik (zit. A. 7) 16.

33 J. Ruysschaert, Les manuscrits corviniens de la Vaticane. Revue française d'histoire du livre N. S. 36 (1982) 289.

34 W. Metzger, Die humanistischen, Triviums- und Reformationshandschriften der Codices Palatini latini in der Vatikanischen Bibliothek (Cod. Pal. lat. 1461-1914) (Kataloge der Universitätsbibliothek Heidelberg 4). Wiesbaden 2002, 146-148, speziell 147.

35 Zum Werk des Lehrbüchermeisters vgl. vor allem G. Sснмпдт, in : Die Gotik in Niederösterreich, Wien $1953,107 \mathrm{f}$.

36 Vgl. A. Haidinger, in: Ausst.-Kat. Die Kuenringer. Wien 1981, Nr. 203.- Die vom Autor geäußerte Vermutung, der in Klosterneuburg im 3. Viertel des 15. Jahrhunderts tätige Regula-Meister könnte auf Grund seines grundsätzlich verwandten Rankenstils aus dem Atelier der in Rede stehenden LadislausHandschriften hervorgegangen sein, wird nicht länger aufrecht gehalten (Vgl. A. Haidinger, Verborgene Schönheit. Die Buchkunst im Stift Klosterneuburg. Klosterneuburg/Wien 1998, 53.).

37 Vgl. u. a. J. Krása, Knižní malířství, in: Pozdně gotické umĕní v Čechách. Prag 1978, 394-396; Haidinger (zit. A. 36); K. Stejskal, P. Voit, Illuminované rukopisy doby husitské. Prag 1990, 63 f., Nr. 53; F. Hoffmann, Soupis rukopisů knihovny kláštera Premonstrátů Teplá (Catalogus codicum manu scriptorum bibliothecae monasterii Teplensis ordinis Praemonstratensis) I. Prag 1999, 435-438 (Nr. 580). 
zusammengesetzt, die wahrscheinlich bereits im 6. Jahrzehnt des 15. Jahrhunderts entstanden $^{38}$. Der Grundstock der Handschrift (Bl. 5-86) wurde vom Melker Professen Johannes von Ulm in Melk wohl vor Abreise des Königs nach Prag geschrieben, im Stift Melk mit zahlreichen Fleuronnéeinitialen ausgeschmückt ${ }^{39}$ und in Melk oder Prag mit Deckfarbeninitialen eines Illuminators versehen, dessen Stil sowohl in österreichischen wie in böhmischen Handschriften nachgewiesen werden kann ${ }^{40}$. Der Rest der Handschrift entstand in Prag; der mit einem Bildnis des Königs auf 93r (Abb. 28) ausgeschmückte zweite Teil (Bl. 87-179) sicher noch zu dessen Lebzeiten.

Zwei Schreiber des Gebetbuches sind auch im Vokabular nachweisbar: Die Haupthand des zweiten Teils, die $87^{\mathrm{r}}-137^{\mathrm{r}}$, Z.4 sowie das verbundene Doppelblatt $143^{\mathrm{r}}-144^{\mathrm{v}}$ des Gebetbuches beschrieben hat ${ }^{41}$, ist ohne Zweifel mit Hand B des Vokabulars, dem Schreiber der Notiz über die Krönung des Ladislaus in Prag gleichzusetzen (vgl. Abb. 3 f. mit Abb. 27-29): Die Händegleichheit wird unter anderem durch die Verwendung identischer ZeilenschlußZeichen (c-ähnliches Zeichen über Punkt) und Kürzungsstriche sowie durch übereinstimmende Majuskelformen belegt. Auch Hand C des Vokabulars, der Schreiber der nachgetragenen Notiz über den Tod des Ladislaus, ist im Gebetbuch vertreten: Ihm sind die Nachträge auf $3^{\mathrm{r}-4^{\mathrm{v}}}$ des Gebetbuches zuzuschreiben ${ }^{42}$ (vgl. Abb. 2 mit Abb. 19-22).

Die Tatsache, daß dem im Vokabular nach dem Tod des Ladislaus tätigen Schreiber (Hand C) auch die Nachträge im Gebetbuch auf $1^{{ }^{r}-4^{v}}$ zuzuweisen sind, legt nahe, daß beide Handschriften nach dem Tode des Königs denselben Besitzer hatten. Da Hand C aus paläographischen Gründen auch für die Inschriften auf dem Devotionsbild des Johannes Holubař auf $3^{v}$ des Vokabulars und wahrscheinlich auch für die nachgetragenen letzten sieben Zeilen desselben verantwortlich zu machen ist, wird dieser Besitzer mit hoher Wahrscheinlichkeit Johannes Holubař gewesen sein (Vgl. Abb. 14, 15, 18). Denkbar wäre, daß Johannes Holubař die Nachträge selbst eingesetzt hat; inwieweit jedoch jemanden mit der Biographie eines Johannes Holubař die Kenntnis einer kalligraphischen Buchschrift zugetraut werden kann, soll hier nicht erörtert werden. (Vgl. aber S. 33 und 42.)

38 Teile des Gebetbuches in chronologischer Reihenfolge: Teil 1 Bl. 5-86, Teil 2 Bl. 87-179, Teil 3 Bl. 180-203, Nachträge auf Bl. 1-4. Teil 1 und 2 entstanden aus inhaltlichen beziehungsweise ikonographischen Gründen (Darstellung des Ladislaus in beiden Teilen) noch zu Lebzeiten des Ladislaus. Die Nachträge auf Bl. $1^{\mathrm{r}}-4^{\mathrm{v}}$ sowie der keinen höherrangigen Buchschmuck aufweisende dritte Teil sind aus paläographischen Gründen wohl bald nach dem Tode des Königs (1457) hinzugefügt worden.

39 Zum Fleuronnée vgl. A. Haidinger, Studien zur Buchmalerei in Klosterneuburg und Wien vom späten 14. Jahrhundert bis um 1450. Ungedruckte Dissertation. Wien 1980, 100 u. ö.

40 Der Deckfarbenschmuck dieses Teils gehört einer im bayerisch-österreichischen Raum entstandenen Gruppe von Handschriften um den vom Autor mit dem Notnamen ,Josefsmeister“ belegten Illuminator an (Vgl. A. Haidinger, Verborgene Schönheit [zit. A. 36] 49). - Zu stilistisch verwandten böhmischen Handschriften vgl. A. 49.

${ }^{41}$ Trotz weitgehend identischer Minuskelformen ist es durch Beachtung der Zeilenschluß-Zeichen, der Form der Kürzungsstriche und der Majuskeln möglich, eindeutig zwei Hände zu unterscheiden. Das verbunde Doppelblatt 143/144 folgte ursprünglich auf Bl. 133.

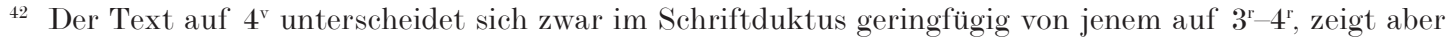
dieselben charakteristischen Kürzungszeichen. Die das Gebet auf $4^{v}$ abschließende Invokation in tschechischer Sprache steht in ihrem kalligraphischen Duktus der Schrift auf $3^{r}-4^{r}$ näher als dem unmittelbar vorangehenden Text auf $4^{\mathrm{v}}$; man beachte etwa die auch im ersten Gebet und in der letzten Textzeile des Vokabulars (Abb. 1, 8) nachweisbaren langen s mit oben angesetztem rechtsgerichteten Dorn. 
Die beiden Ladislaus-Handschriften haben nicht nur eine gemeinsame Geschichte, sondern bereits an ihrer Herstellung haben teilweise dieselben Kräfte mitgewirkt: Sowohl Florator wie Illuminator des Vokabulars waren im zweiten Teil des Gebetbuches tätig ${ }^{43}$. Von den zahlreichen Übereinstimmungen der Fleuronnéeinitialen seien die an den Fadenausläufern ansetzenden, kontinuierlich kleiner werdenden, gequetscht wirkenden Perlenreihen sowie die übereinstimmenden Formen der H-ähnlichen beziehungsweise mäanderförmigen Ausläufer genannt (vgl. Abb. 25 mit 29 bzw. Abb. 26 mit 30). Die kleinteiligen rundlappigen und sticheligen Blattformen der in Deckfarben ausgeführten Ranke auf $3^{\mathrm{v}}$ des Vokabulars begegnen im Gebetbuch vor allem auf 90 und 93 ${ }^{\mathrm{r}}$ (Vgl. Abb. 6 mit Abb. 27 f.); Engel und König auf $93^{\mathrm{r}}$ des Gebetbuches zeigen dieselbe spitzovale Physiognomie wie König Ladislaus im Vokabular (vgl. Abb. 28 mit Abb. 6). Auf die stilistische Verwandtschaft des Deckfarbenschmucks des Gebetbuches mit jenem der 1440 datierten Schellenberg-Bibel (Prag Strahov, Cod. DG III 15) wurde bereits von Stejskal ${ }^{44}$ hingewiesen; die kleinteiligen Blattformen der beiden Ladislaus-Handschriften begegnen jedoch bereits in sehr ähnlicher Form im 1438 entstandenen Brevier des Hanuš von Kolovrat (Prag, Nationalbibliothek, Cod. Osek 71) ${ }^{45}$.

Während die Darstellung des Ladislaus im Vokabular jener im Gebetbuch stilistisch, nicht jedoch ikonographisch entspricht, besteht ein gegenteiliges Verhältnis zur Miniatur eines jugendlichen thronenden Königs im Antiphonar der Brünner Karthäuser Wien, ÖNB, Cod. $1775^{46}$ auf 58v (vgl. Abb. 23). Das Blatt ist mit einer in Deckfarben ausgeführten Initiale zum Responsorium in libris regum und Blattranken auf den Freirändern geschmückt. Im unteren Freirand ist ein thronender König mit einer Gruppe von Kriegern dargestellt, dessen Ähnlichkeit mit Ladislaus bereits von Krása erkannt worden ist ${ }^{47}$, ohne jedoch auf die Miniatur des Vokabulars Bezug zu nehmen.

Die beiden Darstellungen stimmen nicht nur in den Details - etwa im Faltenwurf und in den Konturen des sich am Boden ausbreitenden Gewandes - fast völlig überein ${ }^{48}$, sondern sie haben auch exakt dieselbe Größe (vgl. Abb. 23 f.)! Dies weist auf die Benutzung einer gleichartigen, wenn nicht sogar identischen, eventuell mechanisch kopierten Vorlage zur Anfertigung der Vorzeichnungen hin. Auch Motive der Rankenornamentik des Vokabulars finden sich im Karthäuser-Antiphonar: Man vergleiche etwa die übereinstimmenden Blattmasken an der linken unteren Ecke der Miniatur beziehungsweise an der P-Initiale oder die beiden, die Blattmaske flankierenden Blütenrosetten im linken Freirand des Antiphonars

43 Letzteres enthält eine Vielzahl von ein- bis vierzeiligen Fleuronnéeinitialen; für einen stilistischen Vergleich eignen sich jedoch nur die rund drei Dutzend, in der Regel drei- oder vierzeiligen linksbündigen Initialen.

44 Stejskal (zit. A. 37) 63, Nr. 53.

45 Zum Brevier des Hanuš von Kolovrat vgl. Stejskal (zit. A. 37) 60 f., Nr. 45, Abb. $72-76$.

46 Zur Handschrift vgl. u. a. J. KrásA, Pozdnĕ gotické umení v Čechách. Prag 1978, 409f. beziehungsweise Ausstellungskatalog Od gotiky k renesanci. Výtvarná kultura Moravy a Slezska 1400-1550. II. Brno. Brno 1999, 501 f., Nr. 245 (Katalogbeschreibung K. StejskaL).

47 Vgl. Krísa (zit. A. 46).

48 Die Miniatur des Vokabulars ist etwas sorgfältiger und detailreicher ausgeführt als ihr Pendant im Antiphonar; man vergleiche etwa die Wiedergabe des Kragens, der nur im Vokabular in seinen Details dem Kragen des Königs auf dem Tafelbild des Kunsthistorischen Museums in Wien entspricht. (Vgl. Ausstellungskatalog Friedrich III. Kaiserresidenz Wiener Neustadt. Wiener Neustadt 1966, Nr. 61, Abb. 29.) 
mit der ersten und dritten Rosette im unteren Freirand des Vokabulars (vgl. Abb. 6 mit Abb. 13). Nicht nur der Illuminator des Vokabulars und des zweiten Teiles des Teplaer LadislausGebetbuches ist in der böhmischen Buchmalerei verwurzelt, sondern auch der in diesem Zusammenhang nicht näher zu besprechende Deckfarbenschmuck des in Melk geschriebenen Teils des Ladislaus-Gebetbuches findet sich in böhmischen Handschriften ${ }^{49}$.

Hinsichtlich Schrift- und Federzeichnungsinitialen ergeben sich Parallelen zu den beiden älteren Lehrbüchern Maximilians I. (1459-1519) Wien, ÖNB, Cod. 2368 (um 1465) und Wien, ÖNB, Cod. Ser. n. 2617 (Grundstock 2r-15r ca. 1466/67) ${ }^{50}$. In den Lehrbüchern wird eine große kalligraphische Schrift verwendet, die jener der mit Hand B des Vokabulars gleichgesetzten Haupthand des zweiten Teils des Ladislaus-Gebetbuches engstens verwandt ist. Fichtenau hat auf grundsätzlich Verwandtes in Urkunden der Reichskanzlei aus den frühen vierziger Jahren des 15. Jahrhunderts hingewiesen, die von dem Böhmen Wenzel von Bochow geschrieben worden waren ${ }^{51}$. Daß die „Vorfraktur“ der genannten Lehrbücher von Wolfgang Spitzweg aus diesen Vorbildern entwickelt wurde ${ }^{52}$, ist allerdings angesichts der Tatsache, daß dieser Schrifttyp nicht nur in den rund ein Jahrzehnt jüngeren Handschriften für König Ladislaus sondern bereits in böhmischen Handschriften des vierten Jahrzehnts des 15. Jahrhunderts nachgewiesen werden kann, wenig wahrscheinlich; es werden wohl böhmische Handschriften in der Art des 1438 datierten Breviers des Hanuš von Kolovrat ${ }^{53}$ gewesen sein, die als Vorlage für die Schrift der Maximilian-Lehrbücher dienten. Im Gegensatz zu Böhmen, wo Manuskripte in dieser Schriftart noch im achten Jahrzehnt nachgewiesen werden können $^{54}$, dürfte sich, dem erhaltenen Bestand nach zu urteilen, die „Vorfraktur“ der Lehrbücher als Buchschrift im Wiener Raum nicht durchgesetzt haben. Lediglich im universitären Umfeld scheint sie, vor allem als Auszeichnungsschrift, größere Bedeutung erlangt zu haben ${ }^{55}$.

Auch alle Federzeichnungsinitialen des Vokabulars finden sich in ähnlicher Form in den beiden Zieralphabeten des Lehrbuches Wien, ÖNB, Cod. 2368 ${ }^{56}$. Die Buchstaben des ersten

49 Nach SteJskal (zit. A. 37) hat sich der Illuminator des Melker Gebetbuch-Teils bereits vor 1444 an der Ausschmückung eines tschechischen Marien-Stundenbuches beteiligt und dabei mit dem Meister der Schellenberg-Bibel zusammengearbeitet (A. O. 63 f., Nr. 84). Einen verwandten Falten- und Figurenstil zeigen manche der qualitativ allerdings weit höherwertigen Initialen des Karthäuser-Antiphonars Wien, ÖNB, Cod. 1775 sowie der 1464 datierten, gleichfalls aus dem Kloster Königsfelden bei Brünn stammenden deutschsprachigen Handschrift Wien, ÖNB, Cod. 2828 (vgl. zuletzt Ausstellungs-Katalog Od Gotiky [zit. A. 46], 502 f., Nr. 246.).

50 Zu den Lehrbüchern Maximilians vgl. u. a. H. Fichtendu, Die Lehrbücher Maximilians I. und die Anfänge der Frakturschrift. Hamburg 1961, S. 12 f. sowie A. HaIdinger, Drei Determinations-Ankündigungen aus dem Stift Klosterneuburg. Wiener Jahrbuch für Kunstgeschichte XLVI/XLVII (1993/94) 237-244. In der letztgenannten Publikation wurde in Anmerkung 22 durch ein Versehen ein falscher Aufsatztitel genannt; der entsprechende Verweis bezieht sich auf die oben genannte Publikation Fichtenaus zu den Lehrbüchern Maximilians. Auf Schrift und Musteralphabete des Lehrbuches Wien ÖNB, Cod. 2368 wird der Autor im Kommentarband der für 2004 geplanten Faksimileausgabe dieser Handschrift näher eingehen.

51 Fichtenau (zit. A. 50), $36 \mathrm{f}$.

52 Fichtenau (zit. A. 50), $34 \mathrm{ff}$.

$53 \mathrm{Vgl}$. A. 45.

54 Vgl. z. B. das Orationale des Sigismund Spana (Prag, Nationalbibl., Cod. XIII H 3b). Siehe Abb. 180 in J. KrásA, České illuminované rukopisy 13. - 16. stoleti. Prag 1990.

55 Vgl. Haidinger (zit. A. 50).

56 Fichtenau hat den Hauptschreiber der Lehrbücher mit dem Wiener Neustädter Stadtschreiber Wolfgang Spitzweg gleichgesetzt und ihm auch die Federzeichnungsinitialen der beiden Lehrbücher zugeschrieben. Gleichartige Federzeichnungsinitialen wie in Cod. 2368 finden sich in der jeweils ersten 
Lehrbuch-Alphabets repräsentieren keinen bekannten Initialtypus; in ihrer Grundstruktur entsprechen sie in der Mehrzahl den in Handschriften des 14. und 15. Jahrhunderts häufig anzutreffenden Cadellen. Manche sind teilweise oder zur Gänze aus drapierten Tüchern zusammengesetzt: Dies trifft etwa auf die mittlere O-Initiale auf $22^{\mathrm{v}}$ des Lehrbuches zu (Abb. 31), die der O-Initiale mit dem Jesusknaben auf $1^{\mathrm{r}}$ des Vokabulars entspricht (Abb. 1). Noch deutlicher sind die Ähnlichkeiten zwischen dem Tücheralphabet des Vokabulars und dem zweiten Lehrbuch-Alphabet, dessen Buchstaben gleichfalls aus gedrehten Bändern und Tüchern aufgebaut sind (vgl. Abb. 8 mit 32). Trotz der großen Ähnlichkeiten ist keine direkte Abhängigkeit der beiden Alphabete voneinander festzustellen; einige Buchstaben des Vokabular-Tücheralphabetes (z. B. $K, S, X$ ) zeigen einen komplizierteren Aufbau als ihre Pendants im Lehrbuch.

Das zeitliche Verhältnis der verglichenen Tücheralphabete ist nicht eindeutig. Daß das Tücheralphabet des Vokabulars wohl unmittelbar im Anschluss an die nach dem Tod des Ladislaus († 1457) eingesetzten Federzeichnungen der „Holubař-Stilschicht“ eingetragen worden ist, wurde bereits begründet. Doch auch die Entstehungszeit der Lehrbücher-Alphabete muß nicht gleichzeitig mit der um 1465 erfolgten Anlage der Trägerhandschrift erfolgt sein; der auf ein ursprünglich größeres Format als die Textlagen des Lehrbuches hinweisende starke Beschnitt der Lage mit den Alphabeten sowie die abweichende Qualität des Beschreibstoffs legt nahe, daß sie nicht von Anfang an für das Maximilian-Lehrbuch Wien, ÖNB, Cod. 2368 bestimmt war ${ }^{57}$. Daß die Lehrbücher-Alphabete jedoch nicht wesentlich älter als ihre Trägerhandschrift und wohl im Raum Wiener Neustadt, vielleicht ursprünglich als Musteralphabet einer Buchmalerwerkstätte entstanden sind, wird durch eine in Wiener Neustadt 1458 geschriebene Historienbibel (Hamburg, Staats- und Universitätsbibl., Cod. 8 in scrin.) belegt; sie enthält dutzende qualitätvolle, zweifellos vom Zeichner der LehrbücherAlphabete stammende Federzeichnungs-Initialen ${ }^{58}$.

Ein Novum sind die in Rede stehenden Alphabete freilich nicht. Vergleichbare, wenn auch zumeist weniger kalligraphisch ausgeführte Initialen lassen sich in teils älteren abendländischen Handschriften nachweisen; relativ selten sind jedoch vollständige Musteralphabete überliefert. So etwa in einer 1428 datierten, im süddeutschen oder ostösterreichischen Raum angelegten Lage der Sammelhandschrift München, Universitätsbibliothek, $4^{\circ} \mathrm{Cod}$. ms. $810^{59}$, die auf $45^{\mathrm{v}}-46^{\mathrm{v}}$ einfache, aus Bändern aufgebaute Initialen sowie auf $47^{\mathrm{r}}-48^{\mathrm{v}}$ ein

Schriftspiegelzeile des von Spitzweg geschriebenen Grundstocks des Lehrbuches Wien, ÖNB, Cod. Ser. n. 2617, doch wirken die Initialen des Spitzweg-Textes deutlich unbeholfener als jene der Musteralphabete in Wien, ÖNB, Cod. 2368, so daß die Zuschreibung der Musteralphabete an Spitzweg nicht zu halten ist. Vgl. Fichtenau (zit. A. 50), 34 ff; hingegen Haidinger (zit. A. 50), $241 \mathrm{f}$.

57 Bereits Fichtenau (zit A. 50) weist auf den Wechsel des Pergaments im Lehrbuch hin und folgert daraus, daß die Lage mit den Zieralphabeten „kaum zum ursprünglichen Bestand gehört“ (a. a. O., 18). Er identifiziert den Meister der Alphabete mit dem Schreiber des Hauptteils der beiden Maximilian-Lehrbücher (a. a. O., 21); dagegen Haidinger (zit. A. 50) $241 \mathrm{f}$.

58 Zur Handschrift zuletzt T. Brandis, Die Codices in scrinio der Staats- und Universitätsbibliothek Hamburg (Katalog der Handschriften der Staats- und Universitätsbibliothek Hamburg VII). Hamburg 1972, 39-41; A. K. Нанn, Die Historienbibel Cod. 8 in scrin. der Staats- und Universitätsbibliothek Hamburg (Codices illuminati medii aevi 47). München 1997. Auf die Händegleichheit der zahlreichen Federzeichnungsinitialen der genannten Handschrift und der beiden Musteralphabete des MaximilianLehrbuches Wien, ÖNB, Cod. 2368 wurde bislang nicht hingewiesen; darauf wird an anderer Stelle (vgl. A. 50) näher eingegangen werden.

59 Beschreibung der Handschrift bei M. Reuter, Die Handschriften der Universitätsbibliothek München V: Die lateinischen mittelalterlichen Handschriften aus der Quartreihe. Wiesbaden 2000, 260-269. 
Alphabet aus großen, cadellenähnlichen Majuskeln beherbergt. Lediglich Initialen der letzteren Art finden sich mitunter in größerer Zahl in jüngeren mitteleuropäischen Handschriften $^{60}$, während Tücherinitialen in Handschriften nach 1450 kaum mehr anzutreffen sind.

60 Etwa in der Prager Bibel Wien, ÖNB, Cod. Ser. n. 4742 von 1478 sowie in einem zweibändigen Franziskanergraduale von 1517/1518. Zur erstgenannten Handschrift vgl. HAIDINGER (zit A. 50), 243, zum Graduale (Graz, Zentralbibl. der Wiener Franziskanerprovinz, Cod. A 63/51 beziehungsweise Ljubljana, Franziskanerkloster, Cod. 6772) vgl. N. GoLoB, Kadelne iniciale v dveh volumnih frančiškanskega graduala. Zbornik za umetnostno zgodovino s. n. XXXVIII (Ljubljana 2002) 152-183. 